\title{
Medial Orbitofrontal De-Activation During Tonic Cold Pain Stimulation: A fMRI Study Examining the Opponent-Process Theory
}

This article was published in the following Dove Press journal:

Journal of Pain Research

\author{
Nathalie Bitar ${ }^{1,2}$ \\ Jules R Dugré $\mathbb{B D}^{1,2}$ \\ Serge Marchand ${ }^{3,4}$ \\ Stéphane Potvin (D) ${ }^{1,2}$ \\ 'Research Center of the Institute of \\ Mental Health of Montreal, Montreal, \\ Canada; ${ }^{2}$ Department of Psychiatry, \\ Faculty of Medicine, University of \\ Montreal, Montreal, Canada; ${ }^{3}$ Genome \\ Quebec, Montreal, Canada; ${ }^{4}$ Department \\ of Surgery, Faculty of Medicine, \\ University of Sherbrooke, Sherbrooke, \\ Canada
}

Background: While the concomitant administration of painful and rewarding stimuli tends to reduce the perception of one another, recent evidence shows that pleasant pain relief is experience after the interruption of noxious stimuli. On neurobiological grounds, these opponent processes should translate into decreased activity in brain reward regions during nociceptive stimulation and increased activity in these regions after its interruption. While growing evidence supports the latter assumption, evidence is lacking in humans in support of the former.

Methods: Twenty-six healthy individuals underwent a functional magnetic resonance imaging (fMRI) session during which they were administered a cold pain stimulation, using a novel paradigm which consisted in a cold gel applied on the right foot of participants.

Results: After the interruption of noxious stimulation, participants experienced significant levels of pleasant pain relief. During cold pain stimulation, brain activations were observed in key regions of the pain matrix (eg, thalamus, primary somatosensory cortex and insula). Conversely, the medial orbitofrontal cortex was found to be de-activated. Medial orbitofrontal de-activations were negatively correlated with subclinical pain symptoms.

Discussion: Our results show that a key brain reward region (eg, medial orbitofrontal cortex) is de-activated during cold pain stimulation, a result which is consistent with one of the central assumptions of the opponent-process theory. On methodological grounds, our results show that the cold gel applied to the foot can be used to trigger activations in the pain matrix, and that the interruption of the cold pressor test elicits significant levels of pleasant pain relief. fMRI studies on pain-reward interactions in chronic pain patients are warranted. Keywords: pain, reward, orbitofrontal cortex, fMRI, opponent-process theory, cold pressor test, pain relief, reward

\section{Introduction}

Pain avoidance and reward seeking are two fundamental motivations necessary for survival that are intertwined in complex ways. Historically, several experimental studies have shown that a wide range of pleasant stimuli (eg music, odors, social rewards, humor, drugs) produce analgesic effects in humans. ${ }^{1-4}$ On the other hand, a growing number of experimental studies have shown, more recently, that the cessation of noxious stimulation produces pleasant responses in humans. ${ }^{5-9}$ These latter results are consistent with the opponent-process theory, which postulates that all deviations from homeostasis are accompanied by an opponent process. That is, if a primary sensation (such as a painful one) is abruptly terminated, a sensation of
Correspondence: Stéphane Potvin Research Center of the Institute of Mental Health of Montreal, 733I

Hochelaga, Montreal, Quebec HIN 3V2, Canada

Email stephane.potvin@umontreal.ca 
the opposite valence (eg pleasant) will be felt. ${ }^{5,10}$ Unfortunately, the neurobiological mechanisms involved in the complex pleasure-pain interactions remain poorly understood, especially in humans.

In rodents, seminal work has shown that rewarding drugs such as amphetamines produce anti-hyperalgesic effects, which are abolished by the lesion of mesocorticolimbic dopaminergic neurons, which project from the ventral tegmental area to the nucleus accumbens (eg ventral striatum) and the ventromedial prefrontal cortex. ${ }^{11}$ Likewise, natural rewards (eg odors) have also been shown to produce antinociceptive effects in rodents. ${ }^{12,13}$ In humans, functional neuroimaging studies seeking to investigate the neural pathways involved in pain-reward opponent mechanisms have also focused on rewardinduced analgesia. Indeed, preliminary functional magnetic resonance imaging (fMRI) studies performed in healthy volunteers have shown that the administration of pleasant stimuli (eg music, odors, etc.) reduces the activity of pain-related brain regions (eg the thalamus and somatosensory cortex) elicited by nociceptive stimuli, whereas it seems to increase the activity of brain reward regions (eg ventral striatum). ${ }^{1,3,4}$ By comparison, the mechanisms involved in other key pain-reward opponent processes have attracted less attention until recently. This is the case of two of the core assumptions of the opponentprocess theory, namely that (i) positive experiences should be less pleasurable during nociceptive stimulation and that (ii) positive emotions should be felt after the interruption of a nociceptive stimulation.

On neurobiological grounds, the aforementioned assumptions imply that we should expect to observe (i) decreased activity in brain reward regions during nociceptive stimulation and (ii) increased activity in these regions after its interruption. Thus far, the few fMRI studies investigating the second assumption have produced results consistent with it, since these studies have shown that pain relief is associated with increased activations in the brain reward circuitry, including the nucleus accumbens, the ventral anterior cingulate cortex and the ventromedial prefrontal cortex. ${ }^{14-16}$ By contrast, it has proved more difficult to confirm the first assumption. Indeed, while the fMRI studies from Aharon and colleagues (2006), Becerra and Borsook (2008) and Becerra and colleagues (2013) have highlighted a de-activation of the nucleus accumbens during tonic heat pain stimulation in healthy volunteers, other studies have actually shown the reverse, namely that the activity of the nucleus accumbens and the orbitofrontal cortex was actually increased during the administration of nociceptive stimuli. ${ }^{14,15,17-19}$

Recently, our research team has shown that the administration of the cold pressor test (CPT) applied to a large body surface (up to the shoulder) during a relatively long period of time ( 2 minutes) produces higher levels of pleasant pain relief after the interruption of the nociceptive stimulation, compared to the pleasant responses evoked by the stimulation of a small body surface with a thermode $\left(3 \mathrm{~cm}^{2}\right) .{ }^{20}$ As such, this result implies that the CPT may be better suited to investigate the neural mechanisms involved in pleasure-pain opponent processes, since the procedure produces both spatial and temporal summation. Unfortunately, most fMRI studies using the CPT have used it solely as conditioning stimulus. $^{21,22}$ And when fMRI studies using the CPT have examined pain-elicited activations, most of them did not analyze de-activations. ${ }^{23-25}$ As a result, we are aware of only one fMRI study having used the CPT as its experimental paradigm that has been able to highlight de-activations of brain reward regions (eg amygdala and the ventromedial prefrontal cortex) during cold pain stimulation. ${ }^{26}$ Given the growing interest in pain-reward interactions, these results deserve to be replicated. ${ }^{16,27,28}$

In view of the current state of the literature, the objectives of the current study were (i) to show that the interruption of a nociceptive stimulation using the CPT produces high levels of pleasant pain relief, (ii) to show that brain reward regions are de-activated during the CPT, and (iii) to examine the correlates of brain reward deactivations evoked by noxious stimuli.

\section{Methods}

\section{Participants}

Twenty-six (15 women) healthy subjects were recruited between the ages of 18 and $35(25 \pm 1.12$; mean \pm standard error of the mean (SEM)). Twenty-four participants were right-handed. Recruitment was done via online advertisements (school platforms and kijiji) and through word of mouth. The exclusion criteria were the following: (1) any DSM-V psychiatric disorder; (2) history of chronic pain; (3) centrally acting medication; (4) neurologic disorders; (5) any unstable medical conditions; and (6) fMRI contraindications (eg metal or electronic implant or pregnancy). Urine drug and pregnancy tests were administered before the scanning session. The study was approved by the ethics committee of the Centre de recherche de l'Institut Universitaire en Santé Mentale de Montréal and was conducted in accordance with the 
declaration of Helsinki. All participants signed a detailed consent form.

\section{Clinical Assessments}

The subclinical psychological symptoms of depression, anxiety, anhedonia and pain were evaluated using the Beck depression inventory-II, the State and Trait Anxiety Inventor-State subscale, the Snaith-Hamilton Pleasure Scale, and the Brief Pain Inventory (BPI). ${ }^{29-32}$ The sociodemographic and psychological characteristics of the participants recruited in the study are described in Table 1.

\section{Psychophysical Procedure Inside the Scanner}

The CPT is a widely used technique for the investigation of pain perception in experimental settings. ${ }^{7,33}$ Since the CPT typically uses a bath of water, the potential spill over

Table I Characteristics of Participants

\begin{tabular}{|l|l|}
\hline Characteristics & Statistics \\
\hline Age (mean \pm SEM) & $25 \pm 1.1246$ \\
\hline $\begin{array}{l}\text { Sex (NF, \%) } \\
\text { Male }\end{array}$ & $1 \mathrm{II}(42)$ \\
Female & $15(58)$ \\
\hline Ethnicity (N,\%) & \\
Caucasian & $14(54)$ \\
Afro-American & $4(15)$ \\
Asian & $8(3 \mathrm{I})$ \\
\hline Level of education (\%) & \\
College degree & 58 \\
Bachelor's degree & $3 \mathrm{I}$ \\
Graduate studies & $1 \mathrm{I}$ \\
\hline Employment status (\%) & \\
Employed & 35 \\
No income & 19 \\
Loan or bursary & 23 \\
Others (ie, independent worker, welfare) & 23 \\
\hline Psychological symptoms (mean \pm SEM) & \\
BDI-II & $5.35 \pm 0.08$ \\
STAI-S & $45.54 \pm 0.26$ \\
SHPS & $50 \pm 0.12$ \\
\hline BPI & $1.69 \pm 0.77$ \\
Pain severity & 0.49 \\
Pain interference & \\
\hline
\end{tabular}

Abbreviations: BDI-II, Beck Depression Inventory-II; BPI, Brief Pain Inventory; SEM, standard of the mean; SHPS, Snaith-Hamilton Pleasure Scale; STAI-S, State and Trait Anxiety Inventory-State subscale. of water around expensive machinery such as MRI scanners is a cause of concern, and is therefore less frequently used than other procedures (eg thermode) in functional neuroimaging studies on pain perception. Some research teams have opted for CPT alternatives safer to use in the fMRI scanner, such as bags of ice water or the coldpressor gel test. ${ }^{34,35}$ Here, our research team opted for a modified CPT using gel which allows to hold constant the temperature of the test during a full fMRI run (as verified during pilot testing performed in six participants). More precisely, a gel was prepared using the same protocol as explained by Lapotka and colleagues (2016). ${ }^{35}$ Once the gel was prepared, it was placed into a plastic bag (4 x 11 inches), which was applied on the bridge of the right foot of the participant. Two types of stimuli were used during the experiment: a cold stimulus inducing pain, and a control stimulus inducing no pain. For the paininducing stimulus, the bags of gel were placed into a freezer, and the temperature of the bags (external surface) was $0^{\circ} \mathrm{C}$, and it remained constant during testing. On the other hand, the control stimulus consisted in a bag of gel that was kept at room temperature $\left(\sim 23^{\circ} \mathrm{C}\right)$.

\section{Experimental Design}

During the whole scanning period, the modified CPT (gel) was administered in two separate runs. While a research assistant maintained the bags of gel on the participant's right foot, participants were asked to lie supine fixating a black screen with a white cross in the middle. Each run lasted 3 minutes, and comprised two experimental conditions lasting 45 seconds each, separated by 45 -second rest periods. During the experimental conditions (45s each), either a cold stimulus (Pain block) or a control stimulus (Control block) was applied. The order of presentation of each experimental condition (Pain or Control) changed between each run. The order of each block in each run was counterbalanced between participants in a pseudo-randomized manner. Noteworthy, previous studies on the topic have included a 5-minute time gap between runs to avoid pain sensitization or habituation. $^{5,23}$ Likewise, we integrated a conservative 10minute interval between each run to ensure that no pain sensitization occurred (during this time gap, T1 images were acquired). In the pilot experiment mentioned above, we were also able to verify that a 10-minute delay between administrations is sufficient to avoid pain sensitization, and to verify that a 45 -second delay between blocks was sufficient to prevent a carry-over effect from the pain to the control condition. A new bag for the control stimulus and 


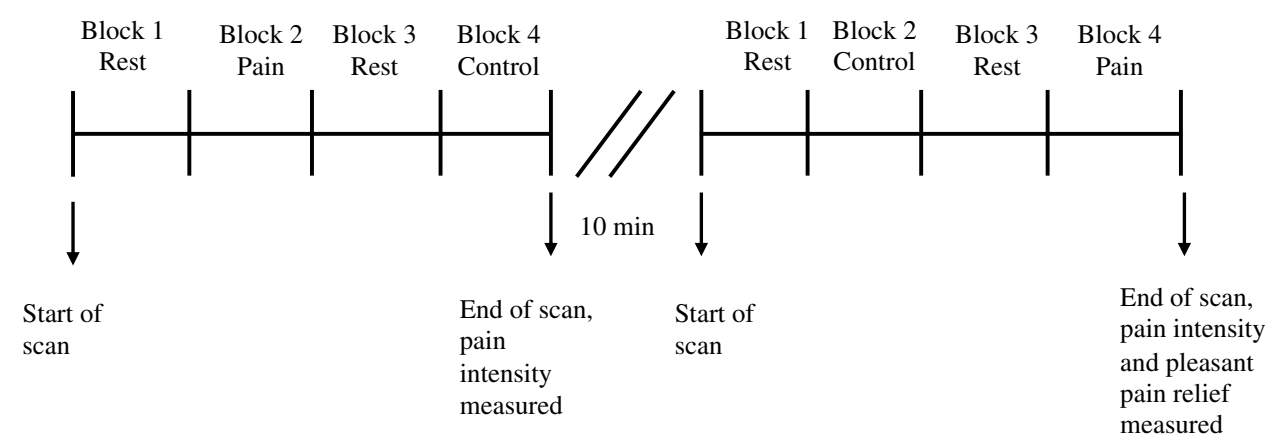

Figure I Experimental design. Each run lasted 3 minutes and was comprised of four 45 second blocks ( 2 experimental conditions and 2 rest periods). There was a 10 minute interval between each run to insure that no pain sensitization occurred. The order of the administration of each stimulus in each run was counterbalanced between each participant in a pseudo-randomized manner.

Abbreviation: fMRI, functional magnetic resonance imagining.

for the pain stimulus was used for each run. Figure 1 provides an illustration of the experimental design. At the end of each run, pain intensity felt during the Cold condition was rated by participants on a verbal scale from 0 (no pain)-100 (maximum tolerable pain), while pleasant pain relief was rated in a scale from 0 (no pleasant pain relief) to 100 (maximum pleasant pain relief) [Note: Pleasant pain relief was only measured in the run where pain was administered at the end].

\section{Psychophysical Procedure Outside the Scanner}

To verify the validity of the modified CPT (gel) administered in the scanner, the CPT was also administered outside the scanner, using a more traditional paradigm. The classic CPT consisted of the immersion of the right arm into a bath of ice water that was kept constant at $10^{\circ} \mathrm{C}$, for a maximum of 2 minutes, by continuously recirculating the water (Julabo F33-HL Heating/refrigerated circulator). The temperature was chosen to be painful yet tolerable for 2 minutes. Using this temperature, our research team showed that the interruption of the CPIT produced significant levels of pleasant pain relief in a previous study. ${ }^{20}$ Participants were instructed to verbally report pain intensity and pain unpleasantness on a scale of 0 to 100 . The measures for pain intensity and pain unpleasantness were taken at the moment the arm was immersed into the bath of cold water and afterwards every 30 seconds, until 120 seconds. With these measures, the mean pain intensity and mean pain unpleasantness were calculated for each participant.

Pleasant pain relief was measured immediately after the CPT (water). In order to explain to participants, the pleasant pain relief phenomenon, we provided an example similar to the one used by Lekness and colleagues
(2013). ${ }^{16}$ Participants were asked to imagine themselves walking in $\mathrm{a}-30^{\circ} \mathrm{C}$ snowstorm for 20 minutes and finally arriving home to feel the warmth of the air inside the house. This warmth would induce the feeling of both pain relief and of pleasure. To fully capture the dynamics of pleasant pain relief, the phenomenon was measured immediately after the end of the immersion, and every 30 seconds afterwards for 4 minutes. Participants were asked to rate their level of pleasant pain relief on a scale of 0 ("I feel relief, but no pleasure") to 100 ("I feel relief and the most intense pleasure possible"). These ratings were used to calculate the mean pleasant pain relief of each participant.

\section{MRI Acquisition Parameters}

Blood oxygenated level dependent signal was acquired on a Prisma Fit 3.0 Teslas scanner from Siemens at the Unité de Neuroimagerie Fonctionnelle de l'Institut de Gériatrie de l'Université de Montréal. Functional images were acquired with a T2-weighted multiband echoplanar imaging (EPI) sequence $\left(\mathrm{TR}=785 \mathrm{~ms} ; \mathrm{TE}=30 \mathrm{~ms} ; \mathrm{FA}=54^{\circ}\right.$; matrix size $64 \times 64$, voxel size $3 \mathrm{~mm}^{3} ; 42$ slices). The functional slices were oriented in transverse plane and were angled to be parallel to the AC-PC line. An inline retrospective motion correction algorithm was employed while the EPI images were acquired. During the same scanning session, highresolution T1-weighted anatomical images were also acquired for each subject $(\mathrm{TR}=2300 \mathrm{~ms}$; $\mathrm{TE}=2.98 \mathrm{~ms}$; $\mathrm{FA}=9^{\circ} ;$ matrix size $=256 \times 256$; voxel size $=1 \mathrm{~mm}^{3} ; 176$ slices .

\section{fMRI Analyses}

fMRI data were preprocessed using the Statistical Parametric Mapping 12 (SPM12) software and the CONN functional connectivity software version $17^{36}$ running in 
Matlab2017a. ${ }^{37,38}$ Functional images were realigned, corrected for motion artifacts with the Artifact Detection Tools implemented in CONN (setting a threshold of $0.9 \mathrm{~mm}$ for subject motion and a global signal threshold of $Z=5$ ), highpass filtered $(0.008 \mathrm{~Hz})$ and co-registered to the corresponding anatomical image. ${ }^{39}$ The anatomical images were segmented (into grey matter, white matter, and cerebrospinal fluid) and normalized to the Montreal Neurological Institute (MNI) stereotaxic space. Functional images were then normalized to MNI stereotaxic space, spatially smoothed with a 3D isotropic Gaussian kernel $(8 \mathrm{~mm}$ fullwidth at half maximum), and resliced to $2 \mathrm{~mm}^{3}$ voxels. For the preprocessing, the anatomical component-based noise correction method, implemented in CONN, was employed as it removes confounding effects from the BOLD time series, such as the physiological noise originating from the white matter and cerebrospinal fluid. The method, therefore, increases validity and sensitivity of analyses. ${ }^{40,41}$

We used a general linear model (GLM) to identify changes associated with pain perception, using a block design. The blocks corresponding to each experimental condition (Pain vs Control) were defined as predictors of interest, which were convolved with the hemodynamic response function. An auto-regressive model was used to account for serial correlations. Predictors of interest were entered as fixed factors in a single-subject GLM, and the parameters of this model were entered into a random-effect model that was used for group analysis using a one-sample $t$-test. Two contrasts were analyzed: [Pain $>$ Control] and [Control $>$ Pain]. The statistical threshold for significance was determined by computing a Monte Carlo simulation, based on Woo, Krishnan and Wager (2014). ${ }^{42,43}$ Assuming a per probability of $\mathrm{p}<0.001$, after 10,000 simulations, a cluster size of 27 resampled voxels was indicated to correct for multiple comparisons at $\mathrm{p}<0.05$. A region of interest analysis was also performed targeting the ventral striatum ( $\mathrm{x}= \pm 14 ; \mathrm{y}=16 ; \mathrm{z}=-4)$, using the small volume correction approach of SPM (corrected $\mathrm{p}<0.05$ ).

As there is evidence that brain activations and deactivations elicited by tonic nociceptive stimuli may vary in the early and late phases of stimulation, we performed sub-analyses on the first and last 22.5 seconds of the Pain blocks that were considered as two epochs. Such secondary analyses were restricted to brain reward regions. ${ }^{17,44}$

\section{Statistical Analysis of Behavioral Data}

Using Pearson's correlations, we tested potential relationships between the following variables: (1) pain intensity and pleasant pain relief, during the administration of the modified CPT (gel); and (2) pain intensity during the modified CPT (gel) and each questionnaire administered (BDI, CAPE, BPI, STAI-S and SNHP). To ensure validity of the modified CPT (gel), we conducted correlation analyses between pain intensity during the original CPT (using a water bath) and pain intensity during the modified CPT, as well as between pleasant pain relief measured after the original CPT and pleasant pain relief measured after the modified CPT. Finally, correlations were conducted between the mean beta of each cluster significantly (de-)activated and; (1) pain intensity and pleasant pain relief (during the modified $\mathrm{CPT}$ ); and (2) each questionnaire administered (STAI, BDI, SNHP, CAPE and BPI). The threshold for statistical significance was set at $\mathrm{p}<0.05$. Statistical analyses were conducted using SPSS, version 25 .

\section{Statistical Power}

The justification of the sample size was based on the results of a previous independent study from our research study that involved 27 participants and showed moderate to high associations between psychophysical and clinical data $(r \geq 0.4 ; \alpha \geq 0.05 ; \beta \geq 80 \%){ }^{20}$

\section{Results \\ Psychophysical Results}

Mean pain intensity during the Pain Blocks of the modified CPT (gel) applied to the right foot was $56.17 \pm 5.45$ while the average pleasant pain relief was $44.5 \pm 5.54$. No participant felt pain during the Control condition.

Pearson's correlation analyses were performed to investigate potential associations between psychophysical measures and subclinical psychological symptoms. Interestingly, a positive correlation was observed between pain intensity during the modified CPT (gel) and pleasant pain relief $(\mathrm{r}=0.602 ; \mathrm{p}=0.001)$. A significant correlation was also found between pain intensity during the modified CPT (gel) and the BPI pain interference subscale $(r=0.477$; $\mathrm{p}=0.014)$. All the correlations with the other clinical scales were non-significant ( $\mathrm{ps}>0.05)$.

\section{Validity Issues}

During the classic CPT (water) administered outside the scanner, the mean pain intensity was $45.92 \pm 4.91$ while the average pleasant pain relief was $42.53 \pm 4.26$. Importantly, a significant correlation was found between pain intensity during the modified CPT (gel) administered during the 
scanning session and pain intensity measured during the CPT (water) administered outside the scanner $(r=0.736$, $\mathrm{p}<0.001)$. Likewise, a positive correlation was observed between pleasant pain relief measured immediately after the scanning session and pleasant pain relief measured after the CPT (water) administered outside the scanner (first measure only; $\mathrm{r}=0.556, \mathrm{p}=0.003$ ).

\section{fMRI Results}

For the [Pain $>$ Control] contrast, four brain regions were found to be significantly activated during the modified CPT (gel), namely the left insula (extending to the left putamen, the left caudate nucleus, the bilateral thalamus and the bilateral insula), the left precuneus (encompassing the left paracentral lobule), the left middle frontal gyrus and the right lingual gyrus (Table 2; Figure 2). The [Control $>$ Pain] contrast revealed a significant deactivation of the right medial orbitofrontal cortex during the administration of the cold pain stimulus (Table 2; Figure 2; Figure 3). Additional analyses revealed that the de-activation in the right medial orbitofrontal cortex was only significant in the late phase of the Pain condition $(\mathrm{x}=8 ; \mathrm{y}=24 ; \mathrm{z}=-10 ; \mathrm{t}=4.57 ; 160$ voxels $)$. Finally, regions of interest analyses revealed no significant activation or de-activation in the ventral striatum.

We found positive correlations between pain intensity during the modified CPT (gel) and activity in the left insula $(\mathrm{r}=0.4 ; \mathrm{p}=0.043$; Figure $4 \mathrm{~A})$ and the left precuneus $(\mathrm{r}=0.54 ; \mathrm{p}=0.004$; Figure 4B). By contrast, brain activity did not correlate with pleasant pain relief. Finally, pain interference as measured with the BPI was found to be positively correlated with activity in the precuneus $(r=0.415 ; p=0.035$; Figure $5 \mathrm{~A})$ and negatively correlated with activity in the right medial orbitofrontal gyrus $(\mathrm{r}=-0.557 ; \mathrm{p}=0.003$; Figure $5 \mathrm{~B})$. No other significant correlations were found between activation clusters and subclinical psychological symptoms.

\section{Discussion}

In view of the mixed findings of fMRI studies investigating pain-reward interactions using noxious heat stimulation with a thermode, we used modified CPT (gel) to induce cold pain in healthy volunteers. Our results showed that the interruption of the nociceptive stimulation caused significant levels of pleasure, as predicted by the opponent-process theory. ${ }^{16}$ As observed in a recent study from our research team, a positive association was found between pain intensity during the CPT and pleasant pain relief experienced after the interruption of the CPT. $^{20}$ The modified CPT (gel) elicited significant activations in the bilateral insula, the bilateral thalamus, the left paracentral lobule, the left middle frontal gyrus and the right lingual gyrus. Importantly, the medial orbitofrontal gyrus was found to be de-activated during cold pain stimulation. Finally, a negative correlation was observed between orbitofrontal activity and pain interference with daily activities.

The most important finding of the current study is the de-activation of medial orbitofrontal cortex that was observed during tonic cold pain stimulation. As such, our

Table 2 Brain Regions (De-)activated During the Modified Cold Pressor Test

\begin{tabular}{|c|c|c|c|c|c|c|c|c|c|}
\hline \multirow[t]{2}{*}{ Contrast } & \multirow[t]{2}{*}{ Brain Region } & \multirow[t]{2}{*}{ L/R } & \multirow[t]{2}{*}{ BA } & \multicolumn{3}{|c|}{ MNI } & \multirow[t]{2}{*}{ Voxel Size } & \multirow[t]{2}{*}{ t-value } & \multirow[t]{2}{*}{$p$ value of Cluster } \\
\hline & & & & $\mathbf{x}$ & y & $\mathbf{z}$ & & & \\
\hline Pain $>c t l$ & $\begin{array}{l}\text { Insula } \\
\text { Putamen } \\
\text { Caudate nucleus } \\
\text { Thalamus } \\
\text { Insula } \\
\text { Precuneus } \\
\text { Paracentral lobule } \\
\text { Middle frontal gyrus } \\
\text { Lingual gyrus }\end{array}$ & $\begin{array}{l}\mathrm{L} \\
\mathrm{L} \\
\mathrm{L} / \mathrm{R} \\
\mathrm{L} / \mathrm{R} \\
\mathrm{L} \\
\mathrm{L} \\
\mathrm{L} \\
\mathrm{R}\end{array}$ & 13 & $\begin{array}{l}-34 \\
-24 \\
-18 \\
-6 \\
-30 \\
-6 \\
-4 \\
-30 \\
18\end{array}$ & $\begin{array}{l}6 \\
4 \\
-26 \\
-26 \\
-26 \\
-42 \\
-28 \\
40 \\
-78\end{array}$ & $\begin{array}{l}10 \\
10 \\
22 \\
12 \\
16 \\
64 \\
72 \\
22 \\
10\end{array}$ & $\begin{array}{l}6349 \\
- \\
- \\
1504 \\
- \\
122 \\
272\end{array}$ & $\begin{array}{l}8.89 \\
8.09 \\
7.28 \\
7.49 \\
6.93 \\
5.54 \\
4.57 \\
4.7 \\
4.47\end{array}$ & $\begin{array}{l}<0.001 \\
<0.001 \\
<0.001 \\
<0.001 \\
<0.001 \\
<0.001\end{array}$ \\
\hline Ctl>pain & Medial orbital & $\mathrm{R}$ & 11 & 6 & 24 & -12 & 129 & 4.38 & $<0.001$ \\
\hline
\end{tabular}

Abbreviations: L, left; R, right; BA, Brodmann area; MNI, Montreal Neurologic Institute. 


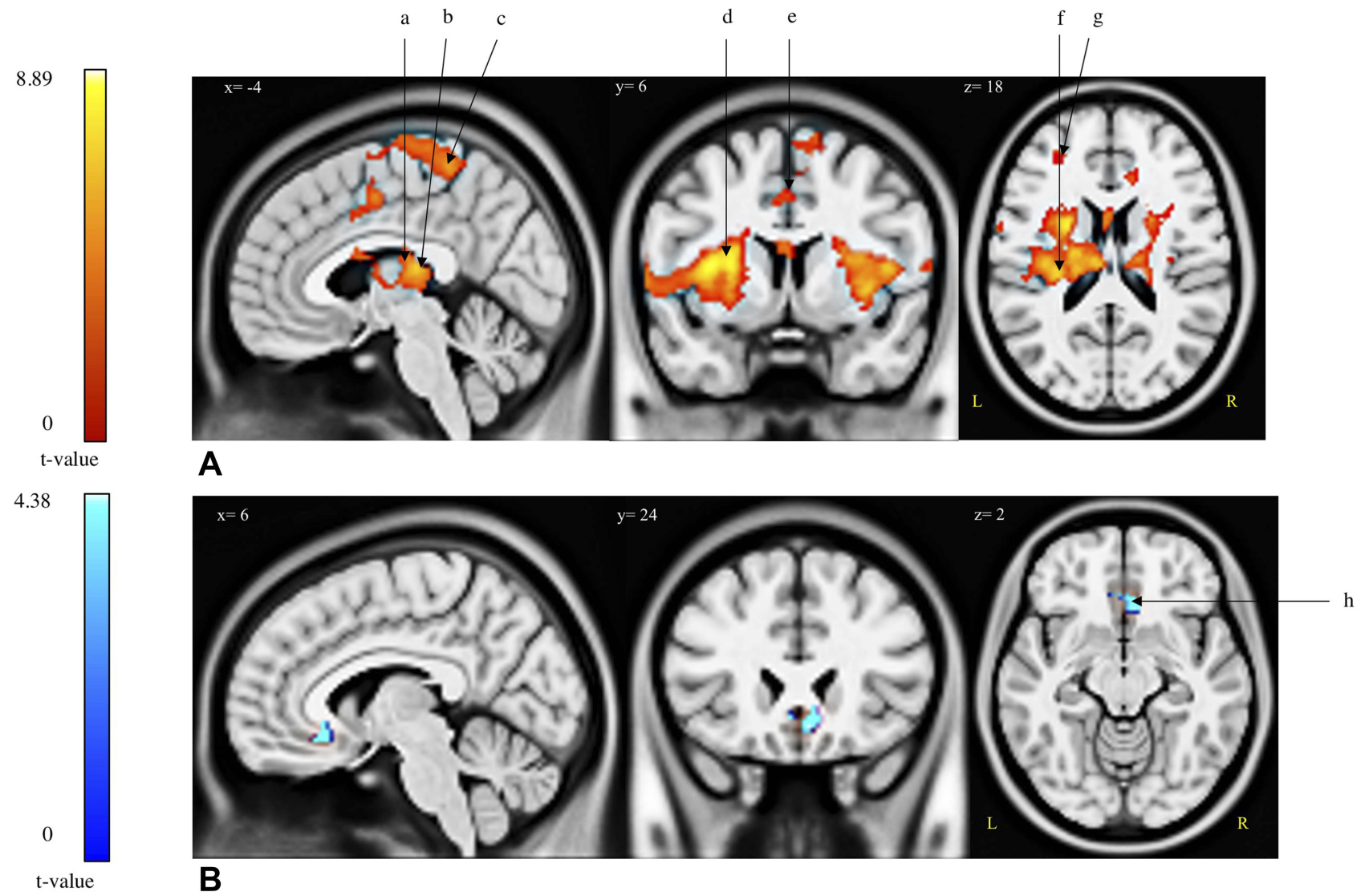

Figure 2 Brain regions (de-)activated during the modified cold pressor test. Two contrasts were analysed: [Pain >Control] and [Control>Pain]. (A) Activations; [Pain $>$ Control] contrast. (B) De-activations; [Control>Pain] contrast.

Abbreviations: a, L-caudate nucleus; b, L/R-thalamus; c, L-paracentral lobule; d, L/R-insula; e, L-precuneus; f, L-putamen; g, L-middle frontal gyrus; h, R-medial orbital.

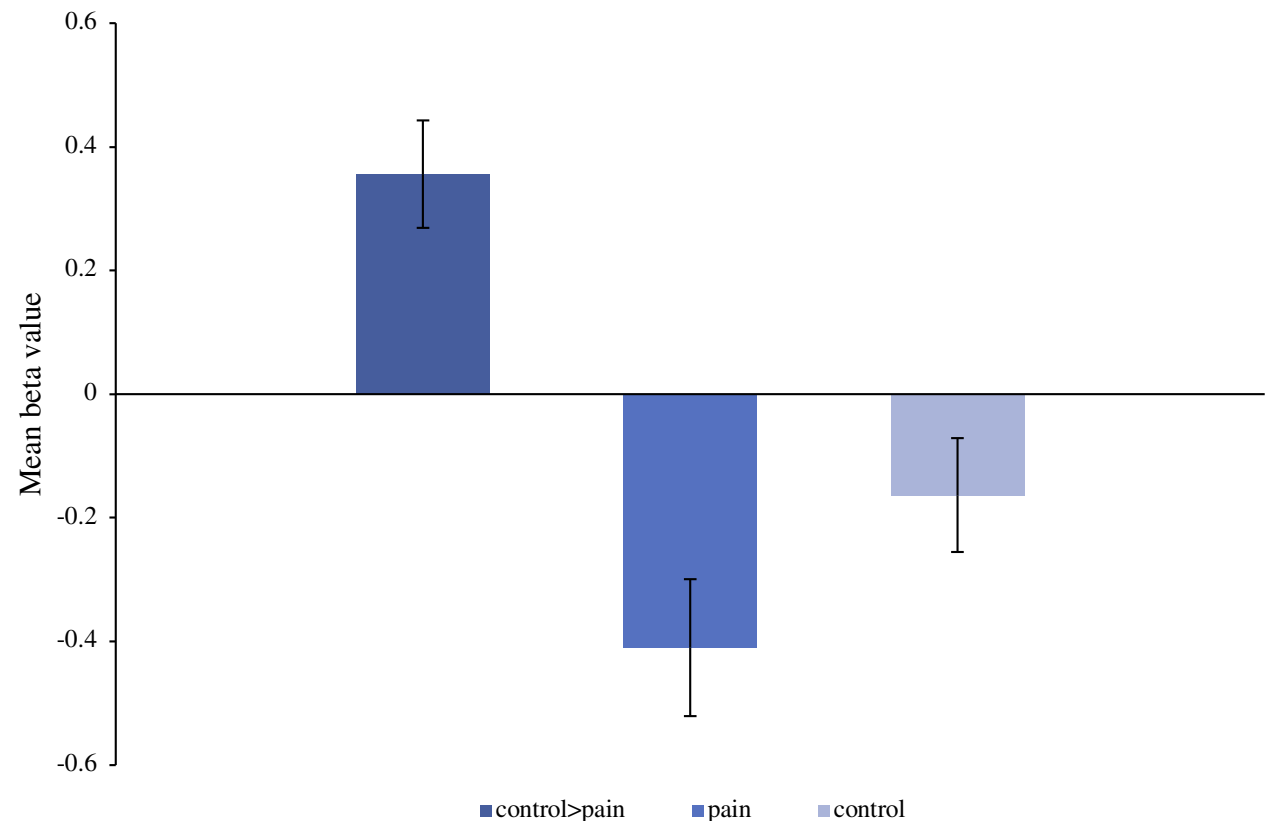

Figure 3 Mean beta. Three mean beta values were extracted for the right medial orbital frontal gyrus in the control>pain contrast. The average beta value for the contrast control >pain was $0.35 \pm 0.09$, the average beta value for this contrast during painful stimulation was $-0.4 \mathrm{I} \pm 0.1 \mathrm{I}$ and during the administration of the control stimulus was $-0.16 \pm 0.09$. These negative values show de-activation in the right medial orbital during both the pain and the control stimuli. However, the de-activation was greater during painful stimulation. Abbreviations: L, left; ctl, control. 

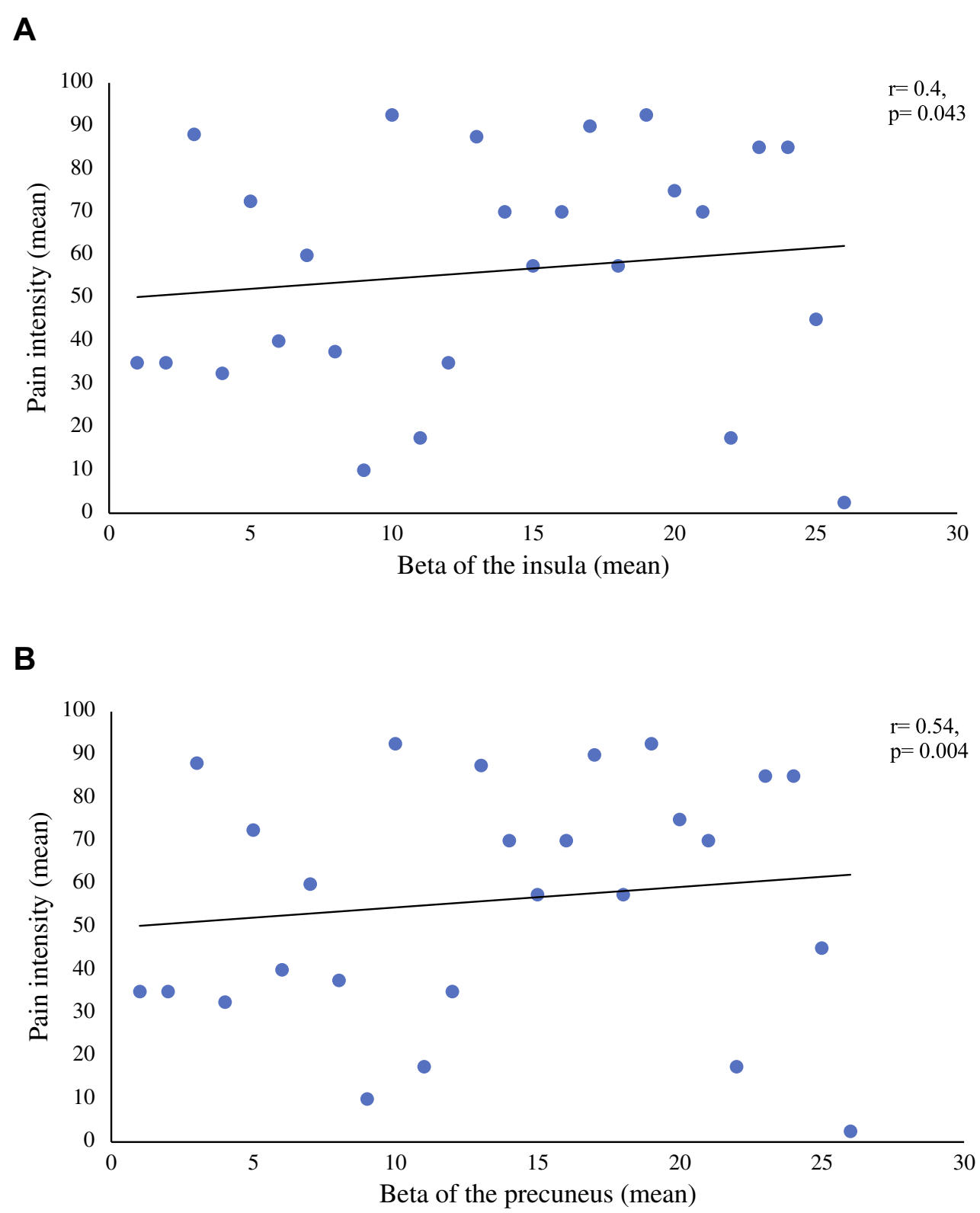

Figure 4 (A) Correlation between the mean beta values of the insula and the mean pain intensity ratings taken during the administration of the modified CPT. This figure illustrates a significative positive correlation between the mean beta values of the insula and the mean pain intensity rating during the administration of both CPT. (B) Correlation between the mean beta values of the precuneus and the mean pain intensity ratings taken during the administration of the modified CPT. This figure illustrates a significative positive correlation between the mean beta values of the precuneus and the mean pain intensity rating during the administration of both CPT.

Abbreviation: CPT, cold pressor test.

main result replicates the finding from Ogino and colleagues (2014), who also observed de-activations of this region during the $\mathrm{CPT}^{26}$ As evidenced by a large metaanalysis involving $206 \mathrm{fMRI}$ studies, the ventromedial prefrontal cortex is one of the two core brain regions involved in reward valuation (the other being the ventral striatum). ${ }^{45}$ Knowing the key role of the medial orbitofrontal cortex in brain reward, our main result lends support to one of the main assumptions of the opponentprocess theory, namely that pain perception should reduce the experience of pleasure, and more precisely, that the activity of brain reward regions should, therefore, be decreased during pain perception. More precisely, we found that the medial orbital de-activation was only significant in late phase of the nociceptive stimuli, suggesting that that brain reward responses to noxious stimuli are dependent on temporal summation. Although this observation must be considered prudently, we also observed that orbitofrontal de-activations were stronger in those who experienced higher levels of pain interference with daily 

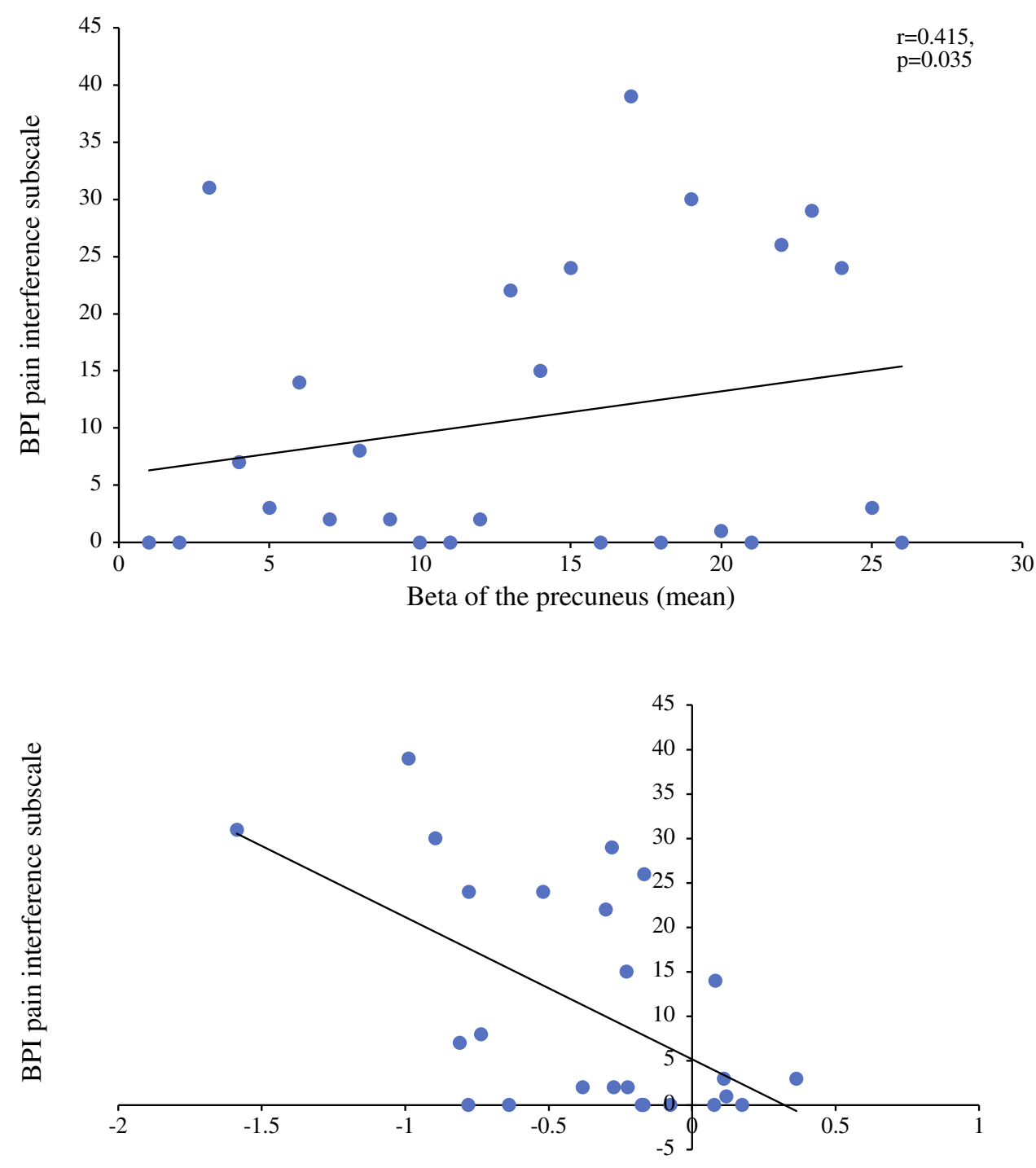

Beta-value of medial orbital frontal gyrus

Figure 5 (A) Correlation between the beta values of the precuneus with the BPI questionnaire (pain interference subscale). This figure illustrates a positive significant correlation between the precuneus during a painful stimulation and the pain interference subscale of the BPI questionnaire. (B) Correlation between the beta values of the medial orbital frontal gyrus with the BPI questionnaire (pain interference subscale). This figure illustrates a negative significant correlation between the medial orbital frontal gyrus during painful stimulation and the pain interference subscale of the BPI questionnaire.

Abbreviation: BPI, Brief Pain Inventory.

activities. In theory, the fact that pain perception induces brain reward alterations may explain why affective conditions like depression and addiction are frequently cooccurrent in those experiencing persistent pain, as recently proposed by Serafini and colleagues (2020). ${ }^{46}$ The depressing effect of nociception on pleasure perception having been studied more often in preclinical than clinical research, it is also important to point out that the main finding of the current study is consistent with reliable findings observed in rodents. Indeed, several preclinical studies have investigated how pain perception modulates brain reward, using the intracranial self-stimulation (ICSS) as the experimental paradigm. In the ICSS procedure, stimulation is delivered via a micro-electrode implanted in the medial forebrain bundle, which connects the ventral tegmental area and the nucleus accumbens (ventral striatum) of the mesolimbic system. Using this paradigm, it has been consistently shown that several nociceptive stimuli, including lactic acid injection, complete Freund's adjuvant injection, paw incision and formalin injection, can all depress brain reward. ${ }^{47,48}$

Noteworthy, in the current investigation, we found no alteration in the activity of the ventral striatum, which is one of the core regions of the brain reward system. ${ }^{11}$ Also 
to consider, the cluster found to be de-activated in the pain condition encompasses the rostral anterior cingulate cortex, which exerts important roles beyond rewarding processing. As such, these observations mean that other alternative or complementary interpretations of our main result need to be considered. As shown by fMRI studies on placebo analgesia, opioid analgesia and stress-induced analgesia, the rostral anterior cingulate cortex plays a key role in endogenous pain modulation. ${ }^{49-51}$ As such, the role of the rostral anterior cingulate cortex in pain inhibition may explain why a negative correlation was observed between the activity in this region and pain interference with daily life. There is also evidence that the rostral anterior cingulate cortex is involved in mind wandering, and that pain perception grasps the attention and attenuates mind wandering propensity. ${ }^{52,53}$ The reduced activity in the rostral anterior cingulate cortex during tonic cold pain perception may thus be interpreted as the inhibition of a key region involved in mind wandering.

Apart from their neurobiological implications, the results of the current fMRI study also have methodological implications. Outside the scanner, the CPT is usually administered using a bath of cold water. Due to water spillage, this method may be problematic for fMRI investigations as the method may cause damage to the equipment in the scanning environment. As an alternative, here, we used cold gel and applied it on the bridge of participants' right foot. Using this method, we observed that cold pain stimulation elicited potent activations in key region of the pain matrix, namely the bilateral insula, the bilateral thalamus and the primary somatosensory cortex (eg paracentral lobule). As such, our results are consistent with previous fMRI studies on pain perception using the CPT, which found consistent activations, during nociceptive stimulation, in the insula, anterior cingulate cortex, the primary sensory cortex and the thalamus. ${ }^{23,25,26,34,54,55}$ Only one other fMRI study on pain perception used specifically the cold gel, which was applied on the left hand, and the study also observed significant pain-elicited activations in the thalamus and insula. ${ }^{24}$ Adding to the validity of our results, we found that pain intensity experienced during the modified CPT (gel) was positively correlated with brain activity in the left insula and the left precuneus. While the anterior and posterior insula are known to be involved in the affective and sensory-discriminative components of pain respectively, growing evidence suggests that the precuneus is involved in the evaluation of the subjective significance of the pain experience. ${ }^{56-59}$
Likewise, we found that the brain activity in the precuneus was positively correlated with the level of interference with daily activities. Taken together, these results strongly suggest that the cold gel is a valid procedure to investigate the neural mechanisms involved in pain perception, using fMRI.

In the current study, we found no association between orbitofrontal de-activations during tonic cold pain stimulation and pleasant pain relief after the interruption of the nociceptive stimulation. One of the potential reasons for the lack of correlation is that subjective pain (pain intensity and pleasant pain relief) was not evaluated continuously inside the scanner, but was evaluated at the end of the run. As such, this methodological limitation may have impeded our chances of detecting significant correlations between neural and subjective measures. However, it is important to mention that the CPT (water) was also administered outside the scanner. By doing so, we found that (i) pain intensity during the modified CPT (gel) correlated with pain intensity during the classic CPT (water), (ii) that pain intensity during the modified CPT (gel) correlated with the level of interference of pain on daily activities, and (iii) that measures of pleasant pain relief taken inside and outside the scanner were intercorrelated. As such, these results suggest that the subjective measures of pain gathered during the modified CPT (gel) are valid. A second limitation of this study is that levels of pain reported on the BPI were low. This was expected since participants recruited in the current study were explicitly excluded if they experienced chronic pain. Still, since the levels of pain reported by participants fell below clinical significance, it implies that the correlations involving the BPI must be interpreted cautiously. A third limitation of our study has to do with the fact that we were not able to standardize the time-of-day of fMRI sessions. There is, however, evidence that pain perception can be influenced by circadian effects. ${ }^{60}$ Finally, another limitation of the study is that it was under-powered to examine sex-differences, although women are at increased risk of developing chronic pain and that sex differences in endogenous pain modulation have been reported. ${ }^{61,62}$

\section{Conclusion}

Using a modified CPT (gel), the results of the current fMRI study showed activations in core regions of the pain matrix, such as the insula, thalamus and primary somatosensory cortex. More importantly, during cold pain 
simulation, significant de-activations were observed in the medial orbitofrontal cortex, one of the core regions of the brain reward system. This result is consistent with one of the central assumptions of the opponent-process theory that had not received enough scientific attention thus far. Future studies will need to identify the potential neurotransmitters involved in the complex pleasure-pain interactions. fMRI studies will also need to examine how pain-reward interactions are involved in the development of chronic pain and its affective comorbidities, namely depression and addiction. To pursue these investigations, the experimental paradigm used in the current study (eg the modified CPT) can be considered as a valid option.

\section{Abbreviations}

CPT, cold pressor test; fMRI, functional magnetic resonance imaging; BPI, Brief Pain Inventory; ICSS, intracranial self-stimulation; GLM, general linear model

\section{Data Sharing Statement}

Genetic data were also collected for this study; therefore, data will not be made available to insure the participants' privacy.

\section{Ethics Approval}

This study received approval from the scientific ethics committee at l'Institut Universitaire en Santé Mentale de Montréal.

\section{Acknowledgments}

SP is holder of the Eli Lilly Canada Chair on schizophrenia research; JD is holder a scholarship from the Fond de Recherche du Québec en Santé.

\section{Funding}

This study was funded by adiscovery grant to SP from the Natural Sciences and Engineering Research Council of Canada.

\section{Disclosure}

The authors declare there is no conflict of interest.

\section{References}

1. Dobek CE, Beynon ME, Bosma RL, Stroman PW. Music modulation of pain perception and pain-related activity in the brain, brain stem, and spinal cord: a functional magnetic resonance imaging study. J Pain. 2014;15(10):1057-1068. doi:10.1016/j.jpain.2014.07.006

2. Dunbar RIM, Baron R, Frangou A, et al. Social laughter is correlated with an elevated pain threshold. Proc R Soc B Biol Sci. 2012;279 (1731):1161-1167. doi:10.1098/rspb.2011.1373
3. Villemure C, Bushnell MC. Mood influences supraspinal pain processing separately from attention. $J$ Neurosci. 2009;29(3):705-715. doi:10.1523/JNEUROSCI.3822-08.2009

4. Younger J, Aron A, Parke S, Chatterjee N, Mackey S. Viewing pictures of a romantic partner reduces experimental pain: involvement of neural reward systems. PLoS One. 2010;5(10):e13309. doi:10.1371/journal.pone.0013309

5. Leknes S, Brooks JCW, Wiech K, Tracey I. Pain relief as an opponent process: a psychophysical investigation. Eur J Neurosci. 2008;28 (4):794-801. doi:10.1111/j.1460-9568.2008.06380.x

6. Leknes S, Lee M, Berna C, Andersson J, Tracey I. Relief as a reward: hedonic and neural responses to safety from pain. PLoS One. 2011;6 (4):e17870. doi:10.1371/journal.pone. 0017870

7. Marchand S, Arsenault P. Spatial summation for pain perception: interaction of inhibitory and excitatory mechanisms. Pain. 2002;95 (3):201-206. doi:10.1016/S0304-3959(01)00399-2

8. Navratilova E, Atcherley CW, Porreca F. Brain circuits encoding reward from pain relief. Trends Neurosci. 2015;38(11):741-750. doi:10.1016/j.tins.2015.09.003

9. Seymour B, O'doherty JP, Koltzenburg M, et al. Opponent appetitive-aversive neural processes underlie predictive learning of pain relief. Nat Neurosci. 2005;8(9):1234. doi:10.1038/nn1527

10. Solomon RL. The opponent-process theory of acquired motivation: the costs of pleasure and the benefits of pain. Am Psychol. 1980;35 (8):691. doi:10.1037/0003-066X.35.8.691

11. Altier N, Stewart J. The role of dopamine in the nucleus accumbens in analgesia. Life Sci. 1999;65(22):2269-2287. doi:10.1016/S00243205(99)00298-2

12. Davies AJ, Kim D, Park J, et al. Hedonic drinking engages a supraspinal inhibition of thermal nociception in adult rats. Pain. 2019;160(5):1059-1069. doi:10.1097/j.pain.0000000000001482

13. Ren K, Blass EM, Zhou QQ, Dubner R. Suckling and sucrose ingestion suppress persistent hyperalgesia and spinal fos expression after forepaw inflammation in infant rats. Proc Natl Acad Sci U S A. 1997;94(4):1471-1475. doi:10.1073/pnas.94.4.1471

14. Becerra L, Borsook D. Signal valence in the nucleus accumbens to pain onset and offset. Eur J Pain. 2008;12(7):866-869. doi:10.1016/j. ejpain.2007.12.007

15. Becerra L, Navratilova E, Porreca F, Borsook D. Analogous responses in the nucleus accumbens and cingulate cortex to pain onset (aversion) and offset (relief) in rats and humans. $J$ Neurophysiol. 2013;110(5):1221-1226. doi:10.1152/jn.00284.2013

16. Leknes S, Berna C, Lee MC, Snyder GD, Biele G, Tracey I. The importance of context: when relative relief renders pain pleasant. PAIN $^{\circledR}$. 2013;154(3):402-410. doi:10.1016/j.pain.2012.11.018

17. Becerra L, Breiter HC, Wise R, Gonzalez RG, Borsook D. Reward circuitry activation by noxious thermal stimuli. Neuron. 2001;32 (5):927-946. doi:10.1016/S0896-6273(01)00533-5

18. Baliki MN, Geha PY, Fields HL, Apkarian AV. Predicting value of pain and analgesia: nucleus accumbens response to noxious stimuli changes in the presence of chronic pain. Neuron. 2010;66 (1):149-160. doi:10.1016/j.neuron.2010.03.002

19. Aharon I, Becerraa L, Chabris CF, Borsooka D. Noxious heat induces fMRI activation in two anatomically distinct clusters within the nucleus accumbens. Neurosci Lett. 2006;392(3):159-164. doi:10.1016/j.neulet.2005.09.054

20. Bitar N, Marchand S, Potvin S. Pleasant pain relief and inhibitory conditioned pain modulation: a psychophysical study. Pain Res Manag. 2018;2018:1-8. doi:10.1155/2018/1935056

21. Potvin S, Marchand S. Pain facilitation and pain inhibition during conditioned pain modulation in fibromyalgia and in healthy controls. Pain. 2016;157(8):1704-1710. doi:10.1097/j.pain.0000000000000573

22. Lewis GN, Rice DA, McNair PJ. Conditioned pain modulation in populations with chronic pain: a systematic review and meta-analysis. J Pain. 2012;13(10):936-944. doi:10.1016/j.jpain. 2012.07.005 
23. La Cesa S, Tinelli E, Toschi N, et al. fMRI pain activation in the periaqueductal gray in healthy volunteers during the cold pressor test. Magn Reson Imaging. 2014;32(3):236-240. doi:10.1016/j. mri.2013.12.003

24. Lapotka M, Ruz M, Salamanca Ballesteros A, Ocón Hernández O. Cold pressor gel test: a safe alternative to the cold pressor test in fMRI. Magn Reson Med. 2017;78(4):1464-1468. doi:10.1002/mrm.26529

25. Macey PM, Wu P, Kumar R, et al. Differential responses of the insular cortex gyri to autonomic challenges. Auton Neurosci. 2012;168(1-2):72-81. doi:10.1016/j.autneu.2012.01.009

26. Ogino Y, Kakeda T, Nakamura K, Saito S. Dehydration enhances pain-evoked activation in the human brain compared with rehydration. Anesth Analg. 2014;118(6):1317-1325. doi:10.1213/ ANE.0b013e3182a $9 \mathrm{~b} 028$

27. Kut E, Candia V, Von Overbeck J, Pok J, Fink D, Folkers G. Pleasurerelated analgesia activates opioid-insensitive circuits. $J$ Neurosci. 2011;31(11):4148-4153. doi:10.1523/JNEUROSCI.3736-10.2011

28. Petrovic P, Kalso E, Petersson KM, Ingvar M. Placebo and opioid analgesia - imaging a shared neuronal network. Science (80-). 2002;295(5560):1737-1740. doi:10.1126/science.1067176

29. Sprinkle SD, Lurie D, Insko SL, et al. Criterion validity, severity cut scores, and test-retest reliability of the Beck Depression Inventory-II in a university counseling center sample. J Couns Psychol. 2002;49 (3):381. doi:10.1037/0022-0167.49.3.381

30. Julian LJ. Measures of anxiety: state-trait anxiety inventory (STAI), beck anxiety inventory (BAI), and hospital anxiety and depression scale-anxiety (HADS-A). Arthritis Care Res. 2011;63(S11):S467S472. doi:10.1002/acr.20561

31. Snaith RP, Hamilton M, Morley S, Humayan A, Hargreaves D, Trigwell P. A scale for the assessment of hedonic tone the SnaithHamilton Pleasure Scale. Br J Psychiatry. 1995;167(1):99-103. doi:10.1192/bjp.167.1.99

32. Wang YP, Gorenstein C. Psychometric properties of the Beck Depression Inventory-II: a comprehensive review. Rev Bras Psiquiatr. 2013;35(4):416-431. doi:10.1590/1516-4446-2012-1048

33. Pud D, Granovsky Y, Yarnitsky D. The methodology of experimentally induced diffuse noxious inhibitory control (DNIC)-like effect in humans. Pain. 2009;144(1-2):16-19. doi:10.1016/j.pain.2009.02.015

34. Sprenger C, Bingel U, Büchel C. Treating pain with pain: supraspinal mechanisms of endogenous analgesia elicited by heterotopic noxious conditioning stimulation. Pain. 2011;152(2):428-439. doi:10.1016/j. pain.2010.11.018

35. Lapotka M, Ruz M, Ballesteros AS, Hernández OO. Cold pressor gel test: a safe alternative to the cold pressor test in fMRI. Magn Reson Imaging Med. 2016;78(4):1464-1468. doi:10.1002/mrm.26529

36. Whitfield-Gabrieli S, Nieto-Castanon A. Conn: a functional connectivity toolbox for correlated and anticorrelated brain networks. Brain Connect. 2012;2(3):125-141. doi:10.1089/brain.2012.0073

37. Ashburner J, Barnes G, Chen C, et al. SPM12 Manual. London, UK.: Wellcome Trust Cent Neuroimaging; 2014

38. Simpson R, Devenyi GA, Jezzard P, Hennessy TJ, Near J. Advanced processing and simulation of MRS data using the FID appliance (FID-A) - an open source, MATLAB-based toolkit. Magn Reson Med. 2017;77(1):23-33. doi:10.1002/mrm.26091

39. Power JD, Barnes KA, Snyder AZ, Schlaggar BL, Petersen SE. Spurious but systematic correlations in functional connectivity MRI networks arise from subject motion. Neuroimage. 2012;59 (3):2142-2154. doi:10.1016/j.neuroimage.2011.10.018

40. Behzadi Y, Restom K, Liau J, Liu TT. A component based noise correction method (CompCor) for BOLD and perfusion based fMRI. Neuroimage. 2007;37(1):90-101. doi:10.1016/j.neuroimage.2007. 04.042

41. Chai XJ, Castañán AN, Öngür D, Whitfield-Gabrieli S. Anticorrelations in resting state networks without global signal regression. Neuroimage. 2012;59(2):1420-1428. doi:10.1016/j. neuroimage.2011.08.048
42. Brown MRG, Benoit JRA, Juhás M, et al. fMRI investigation of response inhibition, emotion, impulsivity, and clinical high-risk behavior in adolescents. Front Syst Neurosci. 2015;9:124. doi:10.3389/ fnsys. 2015.00124

43. Woo C-W, Krishnan A, Wager TD. Cluster-extent based thresholding in fMRI analyses: pitfalls and recommendations. Neuroimage. 2014;91:412-419. doi:10.1016/j.neuroimage.2013.12.058

44. Bogdanov VB, Viganò A, Noirhomme Q, et al. Cerebral responses and role of the prefrontal cortex in conditioned pain modulation: an fMRI study in healthy subjects. Behav Brain Res. 2015;281:187-198. doi:10.1016/j.bbr.2014.11.028

45. Bartra O, Mcguire JT, Kable JW. NeuroImage The valuation system: a coordinate-based meta-analysis of BOLD fMRI experiments examining neural correlates of subjective value. Neuroimage. 2013;76:412-427. doi:10.1016/j.neuroimage.2013.02.063

46. Serafini RA, Pryce KD, Zachariou V. The mesolimbic dopamine system in chronic pain and associated affective comorbidities. Biol Psychiatry. 2020;87(1):64-73. doi:10.1016/j.biopsych.2019.10.018

47. Moerke MJ, Negus SS. Interactions between pain states and opioid reward assessed with intracranial self-stimulation in rats. Neuropharmacology. 2019;160:107689. doi:10.1016/j.neuropharm.2019.107689

48. Negus SS. Expression and treatment of pain-related behavioral depression. Lab Anim. 2013;42(8):292. doi:10.1038/laban.255

49. Bingel U, Lorenz J, Schoell E, Weiller C, Büchel C. Mechanisms of placebo analgesia: $\mathrm{rACC}$ recruitment of a subcortical antinociceptive network. Pain. 2006;120(1-2):8-15. doi:10.1016/j.pain.2005.08.027

50. Bingel $\mathrm{U}$, Wanigasekera $\mathrm{V}$, Wiech $\mathrm{K}$, et al. The effect of treatment expectation on drug efficacy: imaging the analgesic benefit of the opioid remifentanil. Sci Transl Med. 2011;3(70):70ra14. doi:10.1126/ scitranslmed.3001244

51. Yilmaz P, Diers M, Diener S, Rance M, Wessa M, Flor H. Brain correlates of stress-induced analgesia. Pain. 2010;151(2):522-529. doi:10.1016/j.pain.2010.08.016

52. Oshiro Y, Quevedo AS, McHaffie JG, Kraft RA, Coghill RC. Brain mechanisms supporting discrimination of sensory features of pain: a new model. J Neurosci. 2009;29(47):14924-14931. doi:10.1523/ JNEUROSCI.5538-08.2009

53. Kucyi A, Salomons TV, Davis KD. Mind wandering away from pain dynamically engages antinociceptive and default mode brain networks. Proc Natl Acad Sci U S A. 2013;110(46):18692-18697. doi:10.1073/pnas.1312902110

54. Harper RM, Macey PM, Henderson LA, et al. fMRI responses to cold pressor challenges in control and obstructive sleep apnea subjects. J Appl Physiol. 2003;94(4):1583-1595. doi:10.1152/japplphysiol. 00881.2002

55. Richardson HL, Macey PM, Kumar R, Valladares EM, Woo MA, Harper RM. Neural and physiological responses to a cold pressor challenge in healthy adolescents. $J$ Neurosci Res. 2013;91 (12):1618-1627. doi:10.1002/jnr.23283

56. Alshelh Z, Marciszewski KK, Akhter R, et al. Disruption of default mode network dynamics in acute and chronic pain states. NeuroImage Clin. 2018;17:222-231. doi:10.1016/j.nicl.2017.10.019

57. Jensen KB, Regenbogen C, Ohse MC, Frasnelli J, Freiherr J, Lundström JN. Brain activations during pain: a neuroimaging meta-analysis of patients with pain and healthy controls. Pain. 2016;157(6):1279-1286. doi:10.1097/j.pain.0000000000000517

58. Wiech K, Lin CS, Brodersen KH, Bingel U, Ploner M, Tracey I. Anterior insula integrates information about salience into perceptual decisions about pain. J Neurosci. 2010;30(48):16324-16331. doi:10. 1523/JNEUROSCI.2087-10.2010

59. Uddin LQ, Nomi JS, Herbert-Seropian B, Ghaziri J, Boucher O. Structure and function of the human insula. J Clin Neurophysiol. 2017;4(34):1-15.

60. Aviram J, Pud D, Shochat T. Pain sensitivity in healthy young men is modified by time-of-day. Sleep Med. 2013;14(2013):e265. doi:10. 1016/j.sleep.2013.11.645 
61. Gaumond I, Arsenault P, Marchand S. Specificity of female and male sex hormones on excitatory and inhibitory phases of formalin-induced nociceptive responses. Brain Res. 2005;1052(1):105-111. doi:10.1016/ j.brainres.2005.06.011
62. Melchior M, Poisbeau P, Gaumond I, Marchand S. Insights into the mechanisms and the emergence of sex-differences in pain. Neuroscience. 2016;338:63-80. doi:10.1016/j.neuroscience.2016.05. 007

\section{Publish your work in this journal}

The Journal of Pain Research is an international, peer reviewed, open access, online journal that welcomes laboratory and clinical findings in the fields of pain research and the prevention and management of pain. Original research, reviews, symposium reports, hypothesis formation and commentaries are all considered for publication. The manuscript

Submit your manuscript here: https://www.dovepress.com/journal-of-pain-research-journa management system is completely online and includes a very quick and fair peer-review system, which is all easy to use. Visit http:// www.dovepress.com/testimonials.php to read real quotes from published authors. 